\title{
Design and Analysis of the Flexible Auxiliary Bearing Applied to High-speed Magnetic Levitation Equipment
}

\author{
KUN Wang ${ }^{1, a^{*}}$, BAOTIAN Dong ${ }^{1, b}$ and BANGCHENG Han ${ }^{1, c}$
}

${ }^{1}$ Science and Technology on Inertial Laboratory, Fundamental Science on Novel Inertial Instrument \& Navigation System Technology Laboratory, School of Instrument Science and Opto-electronics Engineering, BeiHang University, Beijing, China

awangkunggg@163.com, bdongbaotian@163.com, chanbangcheng@buaa.edu.cn

Keywords: Magnetic bearing, auxiliary bearing, Flexible mechanism, High-speed rotor.

Abstract. To solve the problems of the auxiliary embed in high-speed magnetic levitation equipment, such as can not play the protect role when magnetic bearing out of control, can not absorb the kinetic energy of high-speed rotor and low mechanical precision, a new kind of auxiliary bearing based on flexible mechanism was proposed in this paper. This flexible auxiliary bearing can make big deformation and absorb more rotor kinetic energy than the traditional ball bearing in the same size. The modal and the effect of the flexible auxiliary bearing were analyzed by the FEM method. And the result can meet the design. The conclusions were given in the end.

\section{Introduction}

Over the past decade, due to the rapid advances in magnetic levitation technology, many types of equipment, which are directly driven by high-speed motors, such as blower, compressor, molecular pump, energy storage flywheel and so on, gained rapid development [1]. Because of its high rotation speed and much smaller than traditional motor with same power, magnetic levitation equipment will be found as a useful and safe tool in the field of aerospace, energy storage and precision manufacturing.

Auxiliary bearing is one of the key components in magnetic levitation high-speed equipment. The roles of the auxiliary bearings are mainly as the two follows.

(1) Protect the stator and the rotor.

(2) The role of supporting the rotor.

The role (1) is the key role of the auxiliary bearings. Because the mainly role of the auxiliary bearings is to sustain the impact force of the rotor, the auxiliary bearings are very easily to be broken. Besides, the rotor and the stator might lose their original precision after several changing processes of the auxiliary bearings. Therefore, how to invent a kind of durable auxiliary bearings becomes the bottleneck of the development of the magnetic levitation technology.

There are many thorough and extensive researches about auxiliary bearings were made by Chinese domestic research institutions. Professor Longxiang $\mathrm{Xu}$ and his team had developed a series of improvement measures about the auxiliary bearings in recent years, such as adding an elastic sheet to the outside of the auxiliary bearings[2] and a kind of mechanism that can eliminate the gap of auxiliary bearings when the magnetic bearing lose control[3]. These methods not only protect the rotor and the stator, but also extend the life of the auxiliary bearings.

Minh Nha Pham[4] and his colleagues in Korea also used an elastic sheet to help to prevent the collision between the rotor and the stator. The difference is that they put the elastic sheet in the inner ring of the magnetic bearings. This method equals to add an elastic damping to the auxiliary bearings. However, the installation is more difficult than that of the Professor Longxiang Xu's method. There is also some researchers use the same method to the air bearings [5].

As mentioned above, all the improvement research about the auxiliary bearings is based on the traditional ball bearing. They did not break through the ball bearings birth defect that the thermal expansion of the ball when heating by the friction between high-speed rotor and the ball bearings. The thermal expansion, a dangerous phenomenon, can result in the bearing stuck, which can seriously damage the equipment. In addition, the kinetic energy of the high-speed rotor absorbed by the elastic 
sheet is very limited when the magnetic bearings out of control. It can not completely avoid the ruin of the magnetic bearings. And the other improved structures of the auxiliary bearings are too complex to realize the high mechanical precision.

In this paper, a kind of novel auxiliary bearing based on compliant mechanism was proposed. Because of no balls, the thermal expansion phenomenon is totally eliminated. And it can be made by one processing. So the mechanical precision could be reach very high.

\section{Design}

We proposed a new kind of auxiliary bearing model. The elastic buffer based on compliant mechanism can be used to absorb the kinetic energy of high-speed rotor. The schematic structure is shown in Fig.1. From Fig.1 we can see that the flexible auxiliary bearing can be made by line cutting. And the mechanical precision can be reach very high.

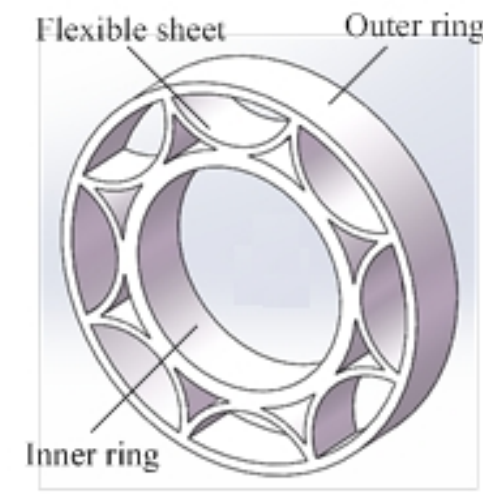

Fig.1 The flexible auxiliary bearing model.

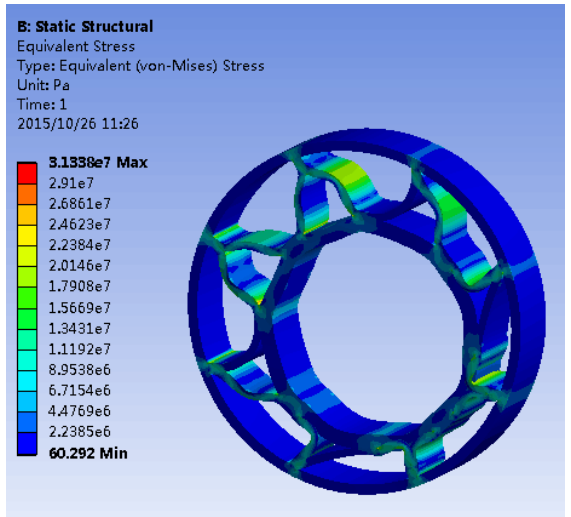

Fig. 2 Stress analysis.

A graphite ring is embedded in the inner ring of the elastic auxiliary bearing to avoid the frictional heat between the high-speed rotor and the elastic auxiliary bearing. Its self-lubricating and low friction coefficient to metal that can greatly reduce the friction make the graphite very suitable as the inner ring of the elastic auxiliary bearing.

When the instability phenomenon happens, the high-speed rotor will repeatedly hit the auxiliary bearings. That means the kinetic energy of the high-speed rotor transfer to the elastic potential energy of the auxiliary bearings. The elasticity of the auxiliary bearing can avoid the rigid impact and the deformation of the high-speed rotor. Also extend the life of the auxiliary bearing and the rotor.

However, certain stiffness is required by the elastic auxiliary bearing. The elastic auxiliary bearing can not deform too much. Or the auxiliary bearing can not play the role of protection for the magnetic bearings. Therefore, the stiffness and the strength become two key points in the design period. Besides, while the instability phenomenon happens, the resonance phenomenon between the high-speed rotor and the elastic auxiliary bearing is not allowed to occur. Consequently, the modal design is another key point in this paper.

\section{Mechanical property analysis}

In this paper, the flexible auxiliary bearing is designed according to the $315 \mathrm{~kW}$ high-speed magnetic bearing motor with $32000 \mathrm{rpm}$. The size of the flexible auxiliary bearing is $\varphi 140 \mathrm{~mm} \times \varphi 80 \mathrm{~mm} \times 26 \mathrm{~mm}$, which is the same as the deep groove ball bearing of GB6216. The material is normal structure steel.

The most basic key point for flexible auxiliary bearing is the mechanical properties such as strength and stiffness. Because the protection role must be based on that the flexible auxiliary bearing can hold the hits and not be destroyed. Therefore, it is necessary to analyze the strength and stiffness of the flexible auxiliary bearing. The $315 \mathrm{~kW}$ high-speed rotor is $60 \mathrm{~kg}$. The impact force between the rotor and the flexible bearing is about 5000N. Fig.2 shows the equivalent stress analysis of the flexible auxiliary bearing. 
From the Fig.2, we can see that the max stress is $31.3 \mathrm{Mpa}$, which is much smaller than the yield strength of $235 \mathrm{Mpa}$. And the safety factor is about 7.5. Fig.2 shows that the designed flexible auxiliary bearing is meeting the engineering requirement in strength.

Fig. 3 shows the deformation of the flexible auxiliary bearing when was hit. From the Fig.3, we can see that the max deformation is $2.24 \mu \mathrm{m}$, which means the stiffness is meeting the engineering requirement.

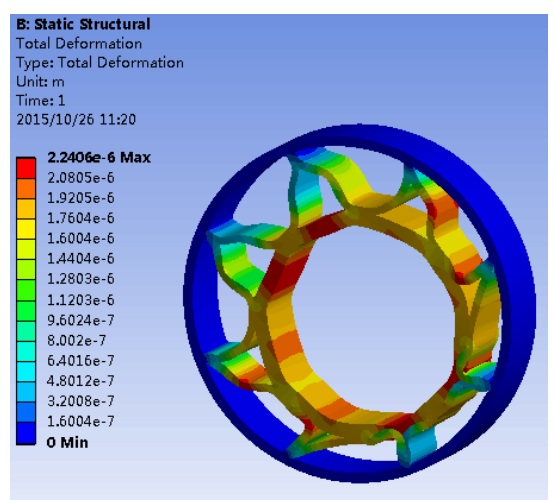

Fig.3 Stiffness analysis.

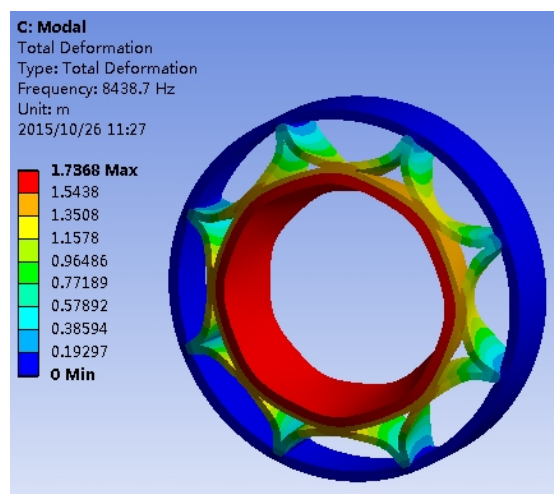

Fig.4 The first modal $(8438.7 \mathrm{~Hz})$.

\section{Modal analysis}

Serious collision can happen between the rotor and the flexible auxiliary bearing. The collision frequency could get up to a few hundred hertz. Therefore, it is necessary to analyze the modal of the flexible auxiliary bearing to avoid the resonance with the rotor. Fig. 4 shows the modal analysis.

From the Fig.4, we can see that the modal of the auxiliary bearing are much far beyond the collision frequency. So it can be concluded that the modal analysis is meeting the engineering requirement.

\section{Comparative analysis}

The auxiliary bearing was designed to be flexible is to make use of the energy absorbing effect of the flexible mechanism. By repeatedly collision, the kinetic energy of the rotor will be constantly changed into the elastic potential energy of the flexible auxiliary bearing. Therefore, the rotation speed can be reduced quickly to avoid unnecessary loss of the equipment. In this section, the comparison of the energy absorption between the flexible auxiliary bearing and the deep groove ball bearing of GB6216 will be analyzed.

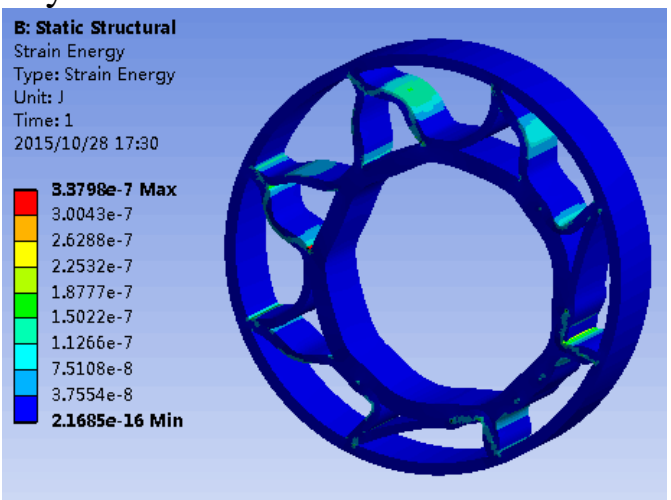

Fig.5 Strain energy of the flexible auxiliary bearing.

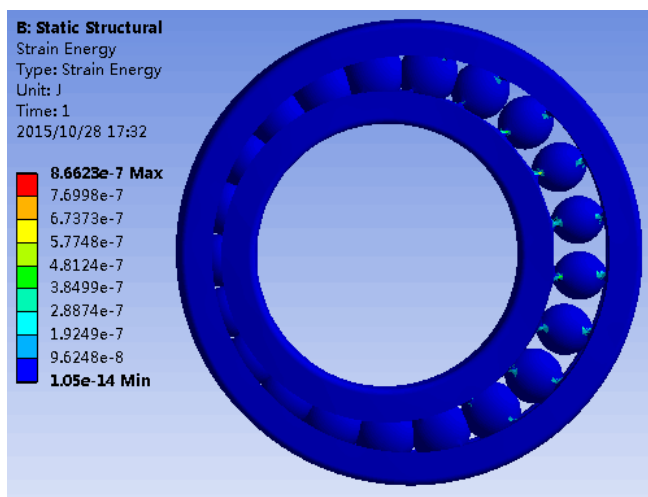

Fig.6 Strain energy of GB6216.

Fig.5 shows the strain energy of the flexible auxiliary bearing. We can see that the max strain energy is $3.38 \mathrm{e}-7 \mathrm{~J}$, which happened at the root area of the elastic sheet. And the whole energy distribution is very even in the elastic sheet.

Fig. 6 shows the strain energy of GB6216. We can see that the max strain energy is 8.66e-7J, which happened only at some balls edge. That means the balls are very easily to be ruined by repeatedly collision. 
Adding all the strain energy of the elements, we can calculate the whole strain energy of the flexible auxiliary bearing and the deep groove ball bearing of GB6216. By only one collision, the whole strain energy of the flexible auxiliary bearing is 4.87e-3J and the whole strain energy of GB6216 is 3.68e-3J. This means the protective effect of the flexible auxiliary bearing is higher $32.3 \%$ than the deep groove ball bearing of GB6216. And the rotor speed can be reduced quicker.

\section{Conclusion}

In this paper, a new kind of auxiliary bearing based on flexible mechanism was proposed. The analysis and simulation were executed. We can draw the conclusions as follows.

(1) The structure of the flexible auxiliary bearing is simple and easy to get high mechanical precision. The size can be designed according to the magnetic bearing equipment. So it breaks the limit of that the auxiliary bearing can only choose from the Standard ball bearings.

(2) The strength and the stiffness of the flexible auxiliary bearing were analyzed by the finite element method. The result can meet the requirement of the magnetic bearing equipment.

(3) The first modal of the flexible auxiliary bearing were analyzed. The result shows that the flexible auxiliary bearing will not resonant with high-speed rotor when the magnetic out of control.

(4) The kinetic energy absorbing effect of the flexible auxiliary bearing and the deep groove ball bearing GB6216 was compared. The result shows that the flexible auxiliary bearing has stronger kinetic energy absorbing effect than that of the bearing GB6216, which is the same size as the flexible auxiliary bearing.

\section{Acknowledgment}

This work was supported by the National Major Project for the Development and Application of Scientific Instrument Equipment of China under Grant 2012YQ040235.

\section{References}

[1] Tang Jiqiang, Xiang Biao, Zhang Yongbin, Dynamic characteristics of the rotor in a magnetically suspended control moment gyroscope with active magnetic bearing and passive magnetic bearing, ISA Transactions, 53 (2014) 1357-1365.

[2] Jin Chaowu, Zhu Yili, Xu Longxiang, Dynamic Analysis of Magnetic Rotor Drop with an Elastic Absorber, Aeroengine, 38 (2012) 12-14.

[3] YU Chengtao, XU Longxiang, JIN Chaowu, Kinematic analysis of Auto-eliminating Clearance Auxiliary Bearing Device for Active Magnetic Bearing Systems, Acta Aeronautica et Astronautica Sinica, 35 (2014) 1-13.

[4] Minh Nha Pham, Hyeong-Joon Ahn, Experimental optimization of a hybrid foil-magnetic bearing to support a flexible rotor, Mechanical Systems and Signal Processing, 46 (2014) 361-372.

[5] I. Iordanoff , B. Bou Said, A. Mezianne, Y. Berthier, Effect of internal friction in the dynamic behavior of aerodynamic foil bearings, Tribology International, 41 (2008) 387-395. 